\title{
EFECTO DE LA MADUREZ, GEOMETRÍA Y PRESIÓN SOBRE LA CINÉTICA DE TRANSFERENCIA DE MASA EN LA DESHIDRATACIÓN OSMÓTICA DE PAPAYA (Carica papaya L., var. Maradol) ${ }^{1}$
}

\author{
Lina Marcela CHAVARRO-CASTRILLÓN², Claudia Isabel OCHOA-MARTÍNEZ ${ }^{2, *}$, Alfredo AYALA-APONTE ${ }^{2}$
}

\section{RESUMEN}

Las papayas obtenidas en el raleo (papayas menos desarrolladas entresacadas de los árboles para beneficiar el crecimiento de las otras) generalmente se descartan. Alternativamente, estas papayas pueden secarse por un proceso de deshidratación osmótica y secado térmico convencional para usarlas como snacks o como ingrediente para otros productos. Se comparó la cinética de transferencia de masa en la deshidratación osmótica de papayas de raleo frente a papayas desarrolladas con diferente grado de madurez, considerando el efecto de la geometría de la muestra y de la presión del proceso. La deshidratación osmótica se efectuó en una solución agitada de sacarosa a $50{ }^{\circ} \mathrm{Brix}$, a $25{ }^{\circ} \mathrm{C}$. Se consideraron tres niveles de madurez: raleo, verde y pintona, tres geometrías: lámina, cilindro y anillo y dos niveles de presión: atmosférica y vacío. Se estudiaron como variables cinéticas la variación de peso (WR), pérdida de agua (WL) y ganancia de sólidos (SG) entre 10 y $180 \mathrm{~min}$. La madurez tuvo efecto significativo sobre las tres respuestas de la cinética a 30 min y sobre SG a $180 \mathrm{~min}$; la geometría tuvo efecto sobre WR y SG a 30 min y sobre los tres parámetros cinéticos a 180 min y la presión solamente tuvo efecto sobre WL y SG a 30 min. La mayor pérdida de agua (65\%) se obtuvo a 30 min para la combinación raleo/cilindro/vacío; mientras que la mayor ganancia de sólidos fue $31 \%$ para el tratamiento verde/lámina/vacío a 180 min. Palabras-claves: deshidratación osmótica, papaya, raleo, pérdida de agua, ganancia sólidos, madurez.

\section{RESUMO}

EFEITO DA MATURIDADE, GEOMETRÍA E PRESSÃO SOBRE A CINÉTICA DE TRANSFERÊNCIA DE MASSA NA DESIDRATAÇÃO OSMÓTICA DE PAPAYA. Os mamões papaya obtidos no "raleo" (mamões papaya menos desenvolvidos entresacadas (desbastados) das árvores, para beneficiar o crescimento dos outros) geralmente se descartam. Alternativamente, estes mamões papaya podem secar-se por um processo de desidratação osmótica e secado térmico convencional, para usá-los como snacks ou como ingrediente para outros produtos. Comparou-se a cinética de transferência de massa na desidratação osmótica de mamões papaya de"raleo" frente a mamões papaya desenvolvidos, com diferente grau de maturidade, considerando o efeito da geometria da mostra e da pressão do processo. A desidratação osmótica se efetuou numa solução agitada de sacarose a $50^{\circ} \mathrm{Brix}$, a $25^{\circ} \mathrm{C}$. Consideraram-se três níveis de maturidade, "raleo", verde e pintona; três geometrias, lâmina, cilindro e anel; e dois níveis de pressão, atmosférica e vazio. Estudaram-se como variáveis cinéticas: a variação de peso (WR), perda de água (WL) e ganho de sólidos (SG) entre 10 e 180 min. A maturidade teve efeito significativo sobre as três respostas a $30 \mathrm{~min}$ e sobre SG a $180 \mathrm{~min}$; a geometria teve efeito sobre WR e SG a $30 \mathrm{~min}$ e sobre os três parâmetros cinéticos a 180 min; e a pressão somente teve efeito sobre WL e SG a 30 min. A maior perda de água (65\%) obteve-se a 30 min para a combinação: "raleo" /cilindro/vazio; enquanto o maior ganho de sólidos foi de $31 \%$ para o tratamento verde/lâmina/ vazio a $180 \mathrm{~min}$.

Palavras-chave: desidratação osmótica, papaya, raleo, perda de água, ganho de sólidos, maturidade.

\section{1 - INTRODUCCIÓN}

Durante la época de crecimiento de las papayas es usual realizar una operación de raleo en los árboles, que consiste en entresacar las papayas menos desarrolladas fisiológicamente para beneficiar el crecimiento de las otras. Estas papayas se descartan y son un problema de disposición de residuos en las zonas agrícolas de producción.

Una alternativa de uso para estas papayas consiste en deshidratarlas osmóticamente y posteriormente someterlas a secado térmico. Esta técnica se ha usado como opción de conservación de los alimentos que presentan contenidos de agua superiores al 85\% [14, 24], para aprovechar los excesos de productos agrícolas que se producen en épocas de cosecha [28], en el desarrollo de nuevos productos atractivos a

${ }^{1}$ Recebido para publicação em 12/7/2005. Aceito para publicação em 6/7/2006 (001573)

${ }^{2}$ Departamento de Ingeniería de Alimentos, Universidad del Valle,

Apartado Aéreo 25360, Cali, Colombia,

E-mail: claochoa@univalle.edu.co

* A quem a correspondência deve ser enviada los consumidores tales como los pasabocas o "snacks" de frutas [31], para suplir las necesidades de materia prima seca como ingrediente para otros productos (yogurt, mermeladas, cereales y productos de panadería) [16] y para disminuir los costos de transporte, de empaque y de almacenamiento. Aunque el secado térmico (secado con aire, microondas, liofilización, fritura) es suficiente para lograr estos objetivos, se ha comprobado que efectuando un tratamiento de deshidratación osmótica (OD) previo al proceso de secado térmico, se reduce el daño de las propiedades texturales, estructurales y sensoriales del alimento [2, 4, 10, 28] y se disminuyen los costos energéticos [12, 26, 29].

La deshidratación osmótica consiste en la eliminación parcial del agua de alimentos sólidos como trozos de frutas y vegetales que se sumergen en una solución concentrada de solutos solubles [5, 20, 33]. Dado que la fase líquida del alimento está separada de la disolución osmótica por las membranas celulares, el equilibrio se logra por el intercambio de agua y de sólidos a través de la membrana [21, 26]. En consecuencia, la OD es un proceso de contra-difusión 
simultáneo de agua y solutos [28] donde ocurren tres tipos de transferencia de masa en contracorriente: flujo de agua del producto a la disolución, transferencia de soluto de la disolución al producto y salida de solutos del producto (azúcares, ácidos orgánicos, minerales y vitaminas que forman parte del sabor, el color y el olor) hacia la disolución [26, 34], este último flujo es muy pequeño comparado con los otros dos, aunque es importante en las características organolépticas del alimento [9, 14].

Aunque se conocen algunas investigaciones de OD sobre papaya madura (con un contenido de sólidos entre 9 y $15{ }^{\circ}$ Brix) $[14,18,25,32]$ y de procesos combinados de OD-congelación [17] y OD-secado con aire [7], ninguno de estos trabajos ha evaluado el comportamiento cinético de la papaya con diferentes grados de madurez, ni tampoco el efecto que tiene la geometría sobre la cinética de OD de la papaya. Es importante mencionar que las papayas de raleo, verdes o pintonas tienen una estructura firme y rígida que permite que aunque las muestras sean delgadas, no se dañen durante el proceso de deshidratación osmótica.

El objetivo de este estudio es comparar la cinética de transferencia de masa en la deshidratación osmótica de papayas de raleo (Carica papaya L., var. Maradol) frente a papayas completamente desarrolladas fisiológicamente con diferente grado de madurez y evaluar el efecto de la geometría de la muestra y de la presión del proceso sobre dicha cinética. Adicionalmente se estudió el comportamiento del cambio de volumen en las muestras deshidratadas.

\section{2 - MATERIALES Y MÉTODOS}

\section{1 - Preparación de las muestras}

Se utilizaron papayas frescas (var. Maradol) de la misma plantación, proporcionadas por fincas ubicadas en el norte del Departamento del Valle del Cauca (Colombia). Se seleccionaron las frutas en tres estados de madurez (papaya de raleo, verde y pintona) de acuerdo al tamaño, al color y al contenido de sólidos solubles ( ${ }^{\circ}$ Brix).

La frutas seleccionadas se lavaron, se pelaron y se cortaron en tres geometrías diferentes: cilindros de $2 \mathrm{~cm}$ de diámetro $\left(7,5 \mathrm{~cm}^{2}\right.$ de área superficial), anillos partidos por la mitad de $3 \mathrm{~cm}$ de diámetro interno y $5 \mathrm{~cm}$ de diámetro externo $\left(15,5 \mathrm{~cm}^{2}\right)$ y láminas de $2 \times 4 \mathrm{~cm}\left(18,4 \mathrm{~cm}^{2}\right)$, todas con un espesor de $2 \mathrm{~mm}$.

\section{2 - Análisis fisicoquímico de muestras frescas}

El contenido de humedad de determinó con el método AOAC, n. 20.013 [1], para el contenido de sólidos solubles se empleó el método AOAC, n. 932.12 [1], la acidez se midió empleando el método AOAC, n. 942.15 [1], el contenido de proteína se determinó con el método AOAC, n. 920.152 [1] y el índice de madurez se calculó a partir de la relación entre el contenido de sólidos solubles y la acidez. La densidad aparente se determinó por el método de desplazamiento de volumen con un picnómetro a $20{ }^{\circ} \mathrm{C}$ utilizando agua destilada como líquido de referencia, y la densidad real se determinó triturando una cantidad considerable de muestra para destruir la estructura y eliminar el aire en su interior, hasta formar una pasta blanda; con esta pasta se llenó el picnómetro (de volumen conocido) y se pesó [3].

\section{3 - Deshidratación osmótica}

Se utilizó una solución de sacarosa de grado alimentario en agua destilada con una concentración de $50{ }^{\circ} \mathrm{Brix}$ a $25^{\circ} \mathrm{C}$. Este soluto tiene amplio uso en el proceso de deshidratación osmótica [7, 16, 22, 25] por su alta masa molar respecto a otros azúcares, lo que reduce la ganancia de sólidos y por lo tanto disminuye el cambio de sabor y aumenta la deshidratación [2, 28, 34].

Las muestras se marcaron con pines y se sumergieron en un recipiente con 4 L de solución osmótica, con una relación fruta: solución de 1:60 para minimizar los cambios de concentración de la solución. La solución se agitó a 480 rpm con un agitador mecánico de pala tipo axial; este agitador crea corrientes que evitan que las muestras floten por su menor densidad respecto a la de la solución. Después de $10,20,30,60,90$ y 180 min de inmersión, se extrajeron las muestras, se enjuagaron en agua destilada para eliminar la solución de la superficie y se secaron con papel absorbente. Posteriormente se determinaron los sólidos solubles usando un refractómetro (ABBE ATAGO $1 \mathrm{~T}$ de Zeiss, termostatado a $20{ }^{\circ} \mathrm{C}$ ), con una precisión de $\pm 0,5{ }^{\circ} \mathrm{Brix}$, el contenido de humedad y la densidad aparente.

Para el proceso con el pulso de vacío, se sumergieron las muestras en la solución osmótica e inmediatamente se introdujeron en el equipo a vacío, hasta alcanzar una presión de 57,6 kPa ( 17 pul Hg), (máximo vacío que permite el equipo que se utilizó), manteniéndose la presión de vacío durante 5 min, después se restituyó la presión y se prosiguió como en los tratamientos a presión atmosférica.

\section{4 - Diseño experimental}

Se realizó un diseño factorial $3 \times 3 \times 2$ completamente al azar. Los tres factores fueron grado de madurez, geometría de la muestra y presión. Los niveles para el grado de madurez fueron papaya de raleo (R), papaya verde (V) y papaya pintona (P); los tres tipos de geometría fueron cilindro (C), anillo (A) y lámina (L) y los niveles para la presión fueron presión atmosférica (OD) y presión de vacío (PVOD).

Se realizaron 18 tratamientos con tres repeticiones por tratamiento, para un total de 54 pruebas. Las variables de respuesta fueron: reducción de peso $(W R)$, pérdida de agua $(W L)$, ganancia de sólidos solubles $(S G)$ y reducción de volumen (VR).

\section{5 - Estudios cinéticos}

Las cinéticas de deshidratación osmótica se analizaron mediante la reducción de peso $(W R)$, pérdida de agua $(W L)$ 
y ganancia de sólidos solubles $(S G)$, usando las ecuaciones (1), (2) y (3), que se obtienen a partir de un balance de masa [4]:

$$
\begin{aligned}
& W R=\frac{M_{t}-M_{0}}{M_{0}} \\
& W L=\frac{\left(M_{t}\right)\left(x_{w, t}\right)-\left(M_{0}\right)\left(x_{w, 0}\right)}{M_{0}} \\
& S G=\frac{\left(M_{t}\right)\left(x_{s, t}\right)-\left(M_{0}\right)\left(x_{s, 0}\right)}{M_{0}}
\end{aligned}
$$

donde:

$M_{0}$ : peso inicial de la muestra (g);

$M_{t}$ : peso de la muestra a un tiempo $t$ de tratamiento (g);

$x_{w, 0}$ : fracción de agua inicial de la muestra;

$x_{w, t}$ : fracción de agua a un tiempo $t$ de tratamiento;

$x_{\mathrm{S}, 0}$ : fracción de sólidos solubles iniciales de la muestra; y

$x_{\mathrm{S}, t}$ : fracción de sólidos solubles a un tiempo $t$ de tratamiento

\section{6 - Análisis estadístico}

Se hicieron análisis de varianza (ANOVA) y pruebas pareadas de Duncan, empleando el programa estadístico Statgraphics Plus 5.1. Se analizaron estadísticamente los resultados que se obtuvieron a 30 y $180 \mathrm{~min}$, teniendo en cuenta que éstos representan las dos etapas claramente identificadas que existen en la OD: deshidratación e impregnación. En la primera, la pérdida de agua es mayor que la ganancia de sólidos, debido a la alta diferencia de concentración entre la solución osmótica y la fase líquida contenida en el interior de la célula de la fruta; y en una segunda etapa, se obtiene una ganancia de sólidos mayor a la pérdida de agua, debido a que la diferencia de concentración ha disminuido [9, 30]. Dependiendo de la etapa en que se encuentre el proceso, los factores involucrados tendrán un efecto diferente sobre las variables de respuesta.

\section{3 - RESULTADOS Y DISCUSIÓN}

\section{1 - Caracterización de la papaya}

En la Tabla 1, se presentan las características de la papaya fresca según su grado de madurez. MÚJICA et al. [18] reporta resultados para papaya var. Maradol similares a los que se presentan para papaya pintona. Todos los resultados son el promedio de tres repeticiones.

\section{2 - Efectos de los factores sobre las cinéticas de transferencia de masa}

En la Figura 1, se observa el comportamiento de la variación de peso, la pérdida de agua y la ganancia de sólidos frente al tiempo para los diferentes factores. En general en todas las gráficas de la Figura 1 se observa mayor perdida de

\begin{tabular}{|c|c|c|c|}
\hline \multirow[t]{2}{*}{ Característica } & \multicolumn{3}{|c|}{ Madurez } \\
\hline & raleo & verde & pintona \\
\hline Contenido de humedad ${ }^{a}$ & $92,22 \pm 0,35$ & $91,41 \pm 0,47$ & $88,63 \pm 0,52$ \\
\hline Sólidos solubles ( ${ }^{\circ}$ Brix) & $5,31 \pm 0,31$ & $6,17 \pm 0,20$ & $9,19 \pm 0,22$ \\
\hline Fibra cruda ${ }^{a}$ & $1,22 \pm 0,06$ & $0,98 \pm 0,06$ & $0,91 \pm 0,06$ \\
\hline Acide $^{b}$ & $0,11 \pm 0,01$ & $0,14 \pm 0,01$ & $0,14 \pm 0,01$ \\
\hline Proteína ${ }^{a}$ & $0,48 \pm 0,01$ & $0,43 \pm 0,01$ & $0,32 \pm 0,01$ \\
\hline Densidad aparente $\left(\mathrm{kg} / \mathrm{m}^{3}\right)$ & $0,93 \pm 0,01$ & $0,93 \pm 0,01$ & $0,94 \pm 0,01$ \\
\hline Densidad real $\left(\mathrm{kg} / \mathrm{m}^{3}\right)$ & $1,04 \pm 0,01$ & $1,03 \pm 0,01$ & $1,03 \pm 0,01$ \\
\hline Porosidad (\%) & $11,36 \pm 0,1$ & $11,08 \pm 0,1$ & $8,80 \pm 0,1$ \\
\hline Índice de madurez & 48,3 & 44,1 & 65,6 \\
\hline
\end{tabular}
agua y de peso en las muestras de papaya de raleo seguidas
TABLA 1 - Caracterización fisicoquímica de la papaya fresca.

por las de papaya verde y papaya madura. Posiblemente este comportamiento se debe a la alta porosidad $(11,36 \%)$ de la papaya de raleo, lo que permite que el agua contenida en el interior de las células salga a través de los poros con mayor facilidad. La ganancia de sólidos fue ligeramente superior para la papaya verde seguida de la papaya de raleo. En la mayoría de los casos, la mayor cantidad de agua se pierde antes de 30 min de inmersión.

Comparando las Figuras 1a, 1c y $1 e$ (OD) se observa, en general, mayor reducción de peso y mayor pérdida de agua para las muestras cortadas en cilindros seguidas por las muestras en anillos y por último las muestras en láminas, mientras que la ganancia de sólidos es menor cuanto menor sea el área superficial (cilindro < anillo < lámina) y al comparar las Figuras $1 b, 1 d$ y $1 f$ (PVOD) se presenta un comportamiento similar. LERICI et al. [11], estudiaron diferentes geometrías y áreas y concluyen que $S G$ aumenta a medida que se aumenta la relación entre el área superficial total (la suma de todas las áreas de las muestras en el proceso) y la longitud característica; para la WL se cumple esta misma premisa solo hasta cierto valor, lo cual hace pensar que existen otros factores involucrados. Igual que en el trabajo de LERICI et al. [11], en este trabajo están mezclados los efectos del área y de la geometría. Puede existir también un efecto, que requiere estudios adicionales, debido al encogimiento de las muestras de acuerdo a sus geometrías. En general, se confirman los resultados que han obtenido otros investigadores en la literatura que han estudiado únicamente el efecto del área utilizando igual geometría y han encontrado que a menor área superficial individual mayor WL [27, 34].

Comparando las Figuras $1 a$ y $1 b$, es claro el efecto que tiene la presión sobre las tres variables de respuesta en los primeros $30 \mathrm{~min}$, pero se observa que ésta no tiene efecto sobre el resultado final, llegando en la mayoría de los casos a valores similares; un efecto análogo se encuentra al comparar las Figuras $1 c$ y $1 d$ y las Figuras $1 e$ y $1 f$, similar a lo obtenido por CORTÉS \& RIAÑO [6], RASTOGI \& RAGHVARAO [23], SERENO et al. [29] y POMPEU et al. [22]. Este comportamiento muestra que se favorece únicamente la velocidad de deshidratación debido a que durante el pulso de vacío se extrae el aire atrapado en los poros de la muestra, y al 
restaurar la presión atmosférica, la solución se incorpora rápidamente al interior del alimento por el intercambio entre el gas o líquido interno en los poros y la solución externa a través de mecanismos hidrodinámicos $[8,18]$ y se aumenta la presión interna de la célula, generándose un gradiente de presión entre el interior y exterior de ésta [13].

Analizando los datos por medio de un diseño categórico de múltiples factores (para factores que no son cuantitativos) y trabajando con un nivel de confiabilidad de $95 \%$, se observa en la Tabla 2, que según el análisis de varianza (ANOVA) para un tiempo de 30 min de deshidratación osmótica, la madurez mostró un efecto significativo sobre los tres factores, la geometría sobre la variación de peso y la ganancia de sólidos y la presión sobre la perdida de agua y la ganancia de sólidos. Para 180 min de inmersión, se observa que la madurez tuvo un efecto significativo en la ganancia de sólidos, la geometría sobre los tres factores y la presión no tuvo efecto significativo a $180 \mathrm{~min}$. Las interacciones no presentaron efecto significativo en ningún caso.
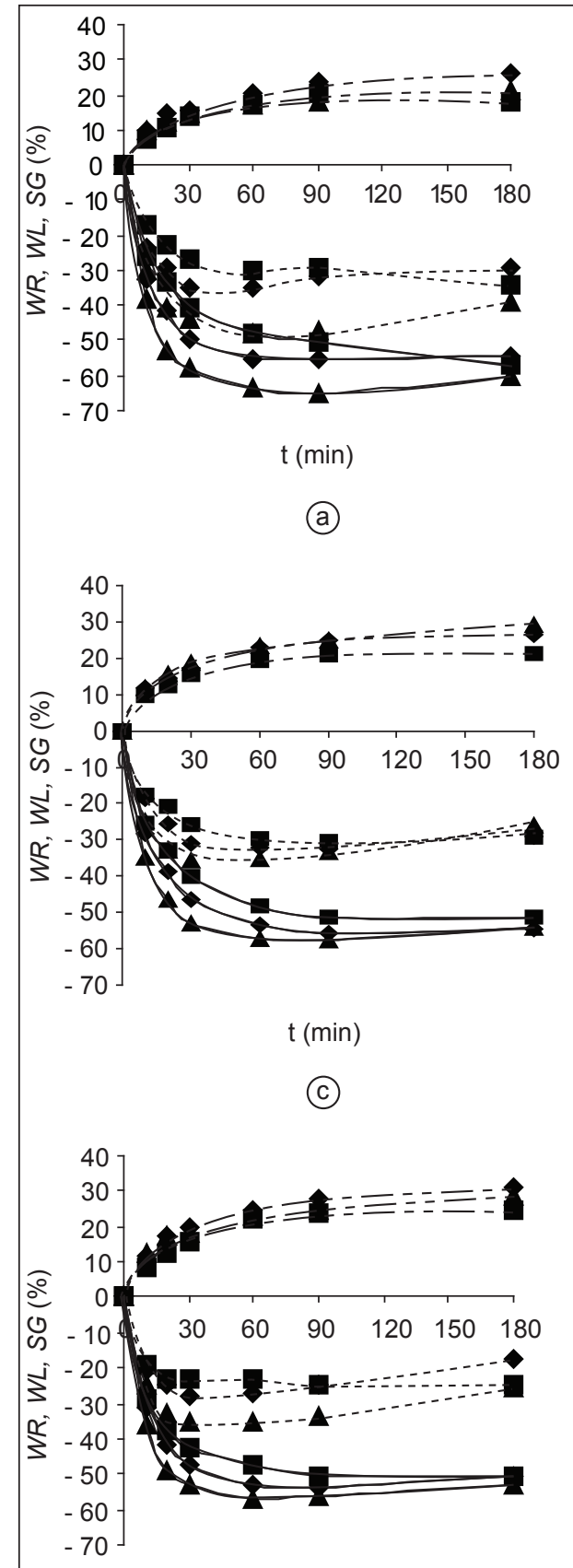

$\mathrm{t}(\min )$ (a)

(c)
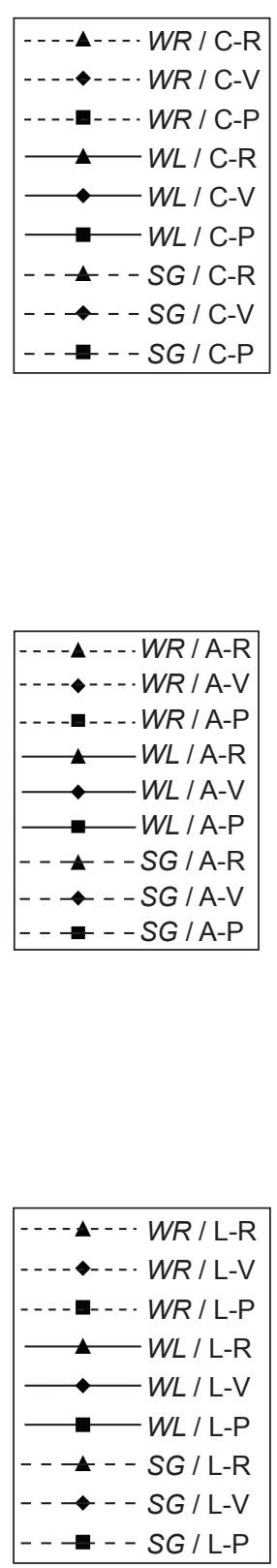

(e)
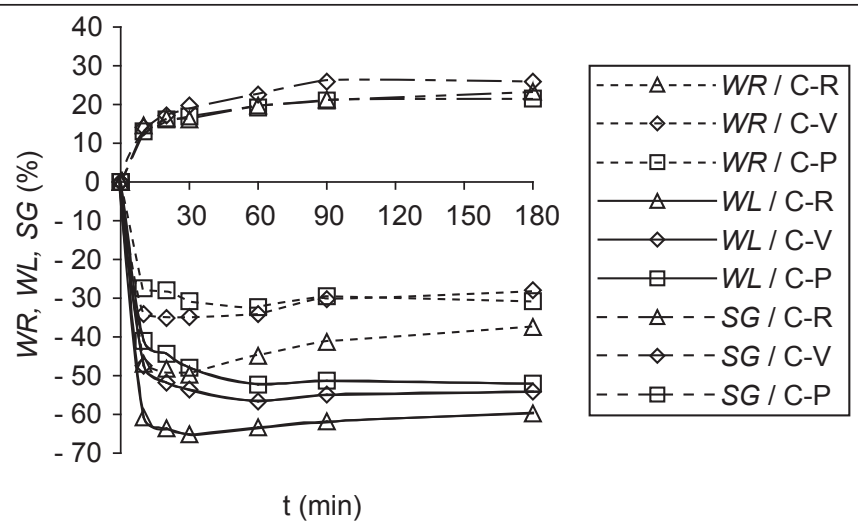

(b)
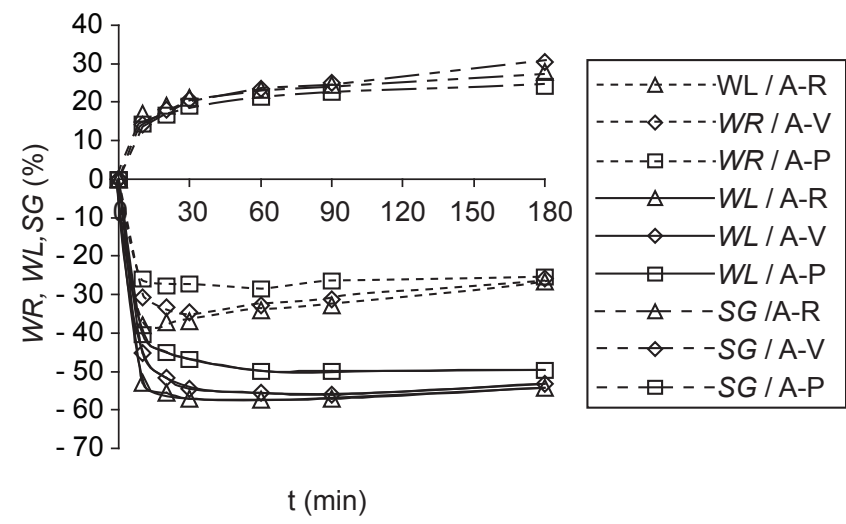

(d)
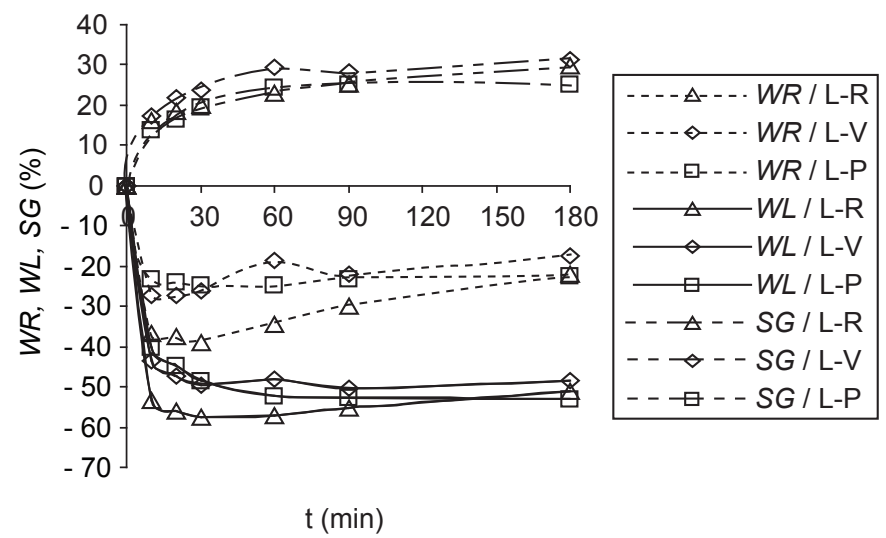

(f)

FIGURA 1 - Influencia del grado de madurez y la geometría sobre las cinéticas en tratamientos a OD (a, c, e) y en tratamientos a PVOD $(b, d, f)$, 
De estos resultados puede concluirse que en la etapa de deshidratación (etapa inicial) la mayoría de los factores tienen efecto significativo en las cinéticas, mientras que en la etapa de impregnación, la geometría es el factor que más influye en los cambios cinéticos.

TABLA 2 - ANOVA para variación de peso, pérdida de agua y ganancia de sólidos a 30 min y 180 min de deshidratación osmótica.

\begin{tabular}{|c|c|c|c|}
\hline & \multirow[t]{2}{*}{$d f$} & \multicolumn{2}{|c|}{ valor de $p$} \\
\hline & & $30 \mathrm{~min}$ & $180 \mathrm{~min}$ \\
\hline \multicolumn{4}{|c|}{ Variación de Peso } \\
\hline \multicolumn{4}{|c|}{ Efectos Principales } \\
\hline A: Madurez & 2 & 0,0000 & 0,2573 \\
\hline B: Geometría & 2 & 0,0228 & 0,0019 \\
\hline C: Presión & 1 & 0,2390 & 0,5446 \\
\hline \multicolumn{4}{|l|}{ Interacciones } \\
\hline$A B$ & 4 & 0,3155 & 0,2775 \\
\hline$A C$ & 2 & 0,9764 & 0,7585 \\
\hline $\mathrm{BC}$ & 2 & 0,8302 & 0,9882 \\
\hline \multicolumn{4}{|c|}{ Perdida de Agua } \\
\hline \multicolumn{4}{|c|}{ Efectos Principales } \\
\hline A: Madurez & 2 & 0,0000 & 0,1267 \\
\hline B: Geometría & 2 & 0,3849 & 0,0248 \\
\hline C: Presión & 1 & 0,0005 & 0,5575 \\
\hline \multicolumn{4}{|l|}{ Interacciones } \\
\hline$A B$ & 4 & 0,3334 & 0,2945 \\
\hline$A C$ & 2 & 0,9074 & 0,9073 \\
\hline $\mathrm{BC}$ & 2 & 0,7297 & 0,9635 \\
\hline \multicolumn{4}{|c|}{ Ganancia de Sólidos } \\
\hline \multicolumn{4}{|c|}{ Efectos Principales } \\
\hline A: Madurez & 2 & 0,0070 & 0,0001 \\
\hline B: Geometría & 2 & 0,0010 & 0,0003 \\
\hline C: Presión & 1 & 0,0000 & 0,2786 \\
\hline \multicolumn{4}{|l|}{ Interacciones } \\
\hline$A B$ & 4 & 0,1857 & 0,2833 \\
\hline$A C$ & 2 & 0,5129 & 0,5081 \\
\hline $\mathrm{BC}$ & 2 & 0,9732 & 0,9198 \\
\hline
\end{tabular}

Los resultados del ANOVA se confirman observando los valores medios de variación de peso, pérdida de agua y ganancia de sólidos que se muestran en la Tabla 3. Se observan las diferencias de las medias de los valores en la pérdida de agua y la ganancia de sólidos entre la presión atmosférica y a vacío para $30 \mathrm{~min}$, las diferencias en los niveles de geometría en la mayoría de los casos, exceptuando para pérdida de agua a $30 \mathrm{~min}$, y las diferencias en las medias entre los distintos grados de madurez con excepción en la variación de peso y la pérdida de agua a $180 \mathrm{~min}$. Por otra parte, observando los valores de las medias generales puede afirmarse que a $30 \mathrm{~min}$ se ha perdido la mayor parte del agua (50\%), mientras que la ganancia de sólidos aumenta considerablemente desde $18 \%$ a 30 min hasta $25 \%$ a $180 \mathrm{~min}$.

Aplicando el procedimiento de comparación múltiple de Duncan, se calcula la diferencia en la media cuadrada mínima (LSM) y se obtiene la diferencia estimada entre cada par de medias de un mismo factor, que son significativamente diferentes de otras. En la Tabla 4 se observa que la principal contribución a los efectos significativos del ANOVA en el factor de geometría es el par de medias cilindro-lámina y en la madurez son los pares pintona-verde y pintona-raleo.

Para un tiempo de 30 min de deshidratación osmótica, no hubo diferencias significativas entre la papaya pintona y la papaya verde en la variación de peso, en la pérdida de agua si hubo diferencias entre los tres grados de madurez y en la ganancia de sólidos el efecto significativo fue causado por las diferencias entre la papaya pintona y la verde. Por otra parte, el efecto de la geometría que se observó en el ANOVA sobre la variación de peso se produjo por las diferencias entre el cilindro y las láminas y sobre la ganancia de sólidos se originó por las diferencias entre los pares cilindro-lámina y cilindro-anillo. Para 180 min de inmersión, el efecto de la madurez en la ganancia de sólidos se provocó por las diferencias entre la papaya pintona y las otras dos y el efecto de la geometría sobre la variación de peso fue causada por diferencias entre las tres geometrías. De estos resultados se puede concluir que no se presentaron mayores diferencias entre la papaya verde y la papaya de raleo excepto para la variación de peso y la pérdida de agua a 30 min y que básicamente hubo diferencias entre las muestras cortadas en cilindros y las cortadas en láminas, en pocos casos los resultados de las cinéticas en anillos fueron diferentes a las láminas y a los cilindros.

\section{3 - Cambios en el volumen y la densidad aparente}

El volumen de las muestras disminuye a medida que transcurre el tiempo del proceso, como se observa en la Figura 2 para los tratamientos OD-C-R y PVOD-C-R (los demás tratamientos no se presentan pero tienen un comportamiento similar); el encogimiento es alto al principio pero al final del proceso se presenta un ligero incremento en el volumen debido a que el material luego de haber estado sometido a tensión (stress) se recupera un poco, absorbiendo una pequeña cantidad de agua [3]. NIETO et al. [19] presentan resultados similares. En general la reducción de volumen $(V R)$ en los tratamientos a PVOD es mayor que a OD hasta $30 \mathrm{~min}$, debido a la disminución de la porosidad inicial de las muestras por la rápida salida de aire asociada al pulso de vacío y el colapso de los poros [3].

En la Figura $3 a$ se observa la correlación lineal relativamente buena que existe entre el encogimiento o pérdida de volumen y la pérdida de agua. Este comportamiento lineal también fue encontrado por AZUARA, BERISTAIN \& GUTIÉRREZ [2], MOREIRA \& SERENO [15] y NIETO et al. [19] en muestras de manzana. Nieto et al. [19] reportaron un coeficiente de correlación de 0,9581. En papaya, MENDOZA \& SCHMALKO [14] correlacionaron linealmente el encogimiento con el contenido de humedad. La Figura $3 b$ muestra un coeficiente de correlación un poco menor entre el encogimiento y la pérdida de peso, pero confirma la relación entre estas dos variables y permite analizar el comportamiento de la densidad aparente. 
TABLA 3 - Medias cuadradas mínimas con intervalo de confianza de 95\% para 30 min y 180 min de deshidratación.

\begin{tabular}{|c|c|c|c|c|c|c|c|}
\hline \multirow[b]{2}{*}{ Nivel } & \multirow[b]{2}{*}{$\begin{array}{l}\text { Número } \\
\text { muestras }\end{array}$} & \multicolumn{2}{|c|}{$W R$} & \multicolumn{2}{|c|}{$W L$} & \multicolumn{2}{|c|}{ SG } \\
\hline & & $30 \mathrm{~min}$ & $180 \mathrm{~min}$ & $30 \mathrm{~min}$ & $180 \mathrm{~min}$ & $30 \mathrm{~min}$ & $180 \mathrm{~min}$ \\
\hline GRAN MEDIA & 54 & $-32,58$ & $-27,36$ & $-50,44$ & $-53,45$ & 18,04 & 25,64 \\
\hline \multicolumn{8}{|l|}{ Madurez } \\
\hline RALEO & 18 & $-40,04$ & $-29,36$ & $-57,12$ & $-55,50$ & 18,13 & 26,62 \\
\hline VERDE & 18 & $-31,14$ & $-24,69$ & $-49,82$ & $-52,63$ & 19,33 & 28,00 \\
\hline PINTONA & 18 & $-26,57$ & $-28,02$ & $-44,37$ & $-52,22$ & 16,66 & 22,28 \\
\hline \multicolumn{8}{|l|}{ Geometría } \\
\hline CILINDRO & 18 & $-36,27$ & $-32,53$ & $-51,92$ & $-55,93$ & 16,24 & 22,77 \\
\hline ANILLO & 18 & $-32,04$ & $-27,96$ & $-49,71$ & $-53,37$ & 18,51 & 25,71 \\
\hline LAMINA & 18 & $-29,43$ & $-21,57$ & $-49,68$ & $-51,05$ & 19,36 & 28,43 \\
\hline \multicolumn{8}{|l|}{ Presión } \\
\hline OD & 27 & $-31,41$ & $-28,07$ & $-47,57$ & $-53,86$ & 16,52 & 25,07 \\
\hline PVOD & 27 & $-33,75$ & $-26,64$ & $-53,31$ & $-53,04$ & 19,55 & 26,20 \\
\hline
\end{tabular}

El error estándar estuvo entre 0.562 y 2.031 .

TABLA 4 - Prueba de rango múltiple para variación de peso, pérdida de agua y ganancia de sólidos para 30 min y 180 min de deshidratación.

\begin{tabular}{|c|c|c|c|c|c|c|}
\hline \multirow[b]{2}{*}{ Método: Duncan 95\% } & \multicolumn{3}{|c|}{$30 \mathrm{~min}$} & \multicolumn{3}{|c|}{$180 \mathrm{~min}$} \\
\hline & WR & $W L$ & SG & $W R$ & $W L$ & SG \\
\hline \multicolumn{7}{|l|}{ Madurez } \\
\hline PINTONA-RALEO & $13,47^{*}$ & $12,74^{*}$ & $-1,46$ & 1,33 & 3,28 & $-4,34^{*}$ \\
\hline PINTONA-VERDE & 4,57 & $5,44^{*}$ & $-2,66^{*}$ & $-3,34$ & 0,41 & $-5,72^{*}$ \\
\hline RALEO-VERDE & $-8,90^{*}$ & $-7,30^{*}$ & $-1,20$ & $-4,67$ & $-2,88$ & $-1,38$ \\
\hline \multicolumn{7}{|l|}{ Geometría } \\
\hline CILINDRO-LAMINA & $-6,84^{*}$ & $-2,24$ & $-3,12^{*}$ & $-10,96^{*}$ & $-4,89^{*}$ & $-5,65^{*}$ \\
\hline CILINDRO-ANILLO & $-4,23$ & $-2,22$ & $-2,27^{*}$ & $-4,57$ & $-2,56$ & $-2,93^{*}$ \\
\hline LAMINA-ANILLO & 2,61 & 0,02 & 0,85 & $6,40^{*}$ & 2,33 & $2,72^{*}$ \\
\hline \multicolumn{7}{|l|}{ Presión } \\
\hline OD-PVOD & 2,34 & $5,74^{*}$ & $-3,03^{*}$ & $-1,43$ & $-0,83$ & $-1,13$ \\
\hline
\end{tabular}

*Denota una diferencia estadística significativa en la media cuadrada mínima (LSM).

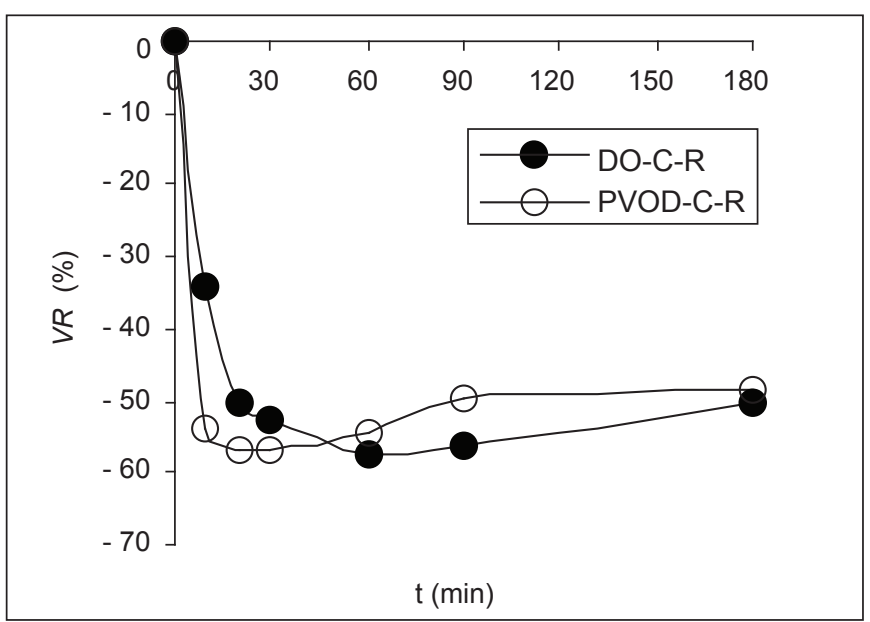

FIGURA 2 - Cambio de volumen en función del tiempo para diferentes condiciones.

De manera inversa a lo que ocurre con el volumen, la densidad aparente de las muestras aumentó con el tiempo, como se puede apreciar en la Figura 4 (los demás tratamientos no se presentan pero tienen un comportamiento similar). De acuerdo a la correlación de la Figura $3 b$, el porcentaje de encogimiento de las muestras es mayor al porcentaje de reducción en el peso, lo que hace que la densidad aparente aumente con el tiempo, similares resultados encontraron AZUARA, BERISTAIN \& GUTIÉRREZ [2]. Hacia el final del proceso, el valor de la densidad aparente mostró un incremento adicional; similar a lo obtenido por NIETO et al. [19]. Según observaciones microscópicas realizadas por NIETO et al. [19], el colapso inicial del tejido producido por la fuerte deshidratación y la recuperación posterior de la forma de las células y los espacios intercelulares explican, por lo menos parcialmente las fluctuaciones de la densidad aparente.

\section{4 - CONCLUSIONES}

La papaya que se obtiene en la operación de raleo presentó la mayor variación de peso y pérdida de agua, posiblemente debido a su alto valor de porosidad y la ganancia de sólidos fue ligeramente menor a la de la papaya verde. Por lo tanto, este tipo de papaya es potencialmente útil deshidratada osmóticamente para utilizarse como ingrediente para otros productos de frutas o someterse a un secado térmico posterior para consumo como pasaboca (snack). En este último caso deberían hacerse pruebas adicionales. La selección de la geometría depende de las necesidades de pérdida de agua y ganancia de sólidos, de acuerdo al tipo de secado térmico que se desee realizar posteriormente. 

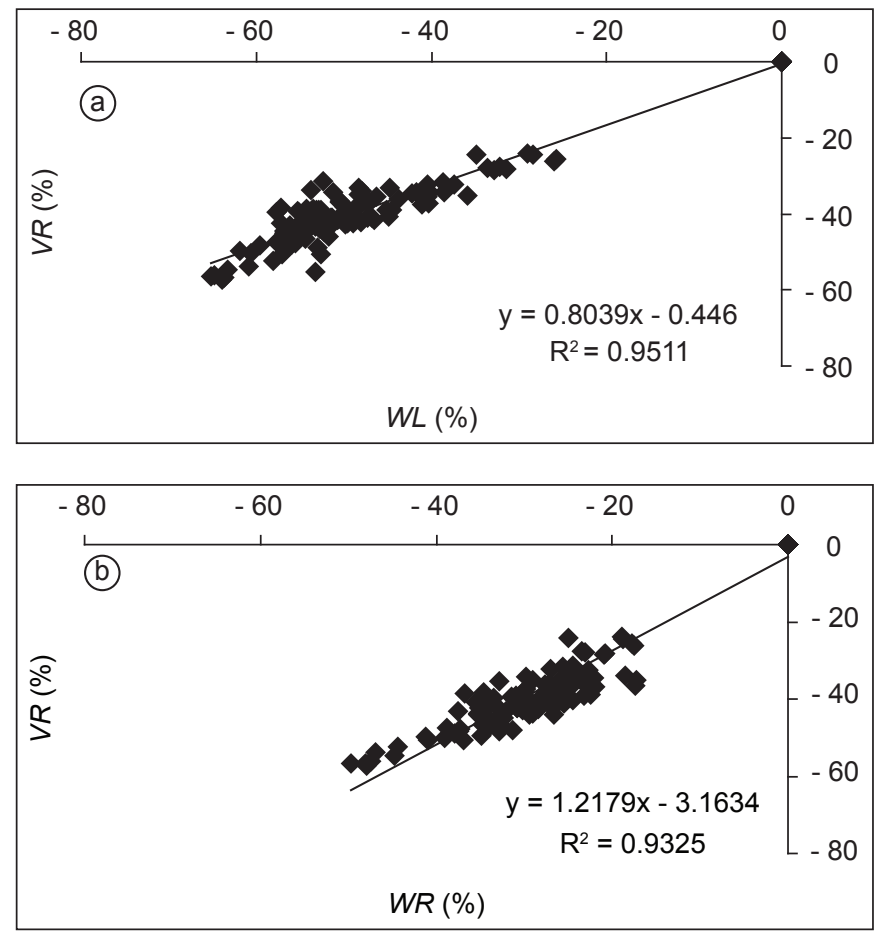

FIGURA 3 - Relación entre el encogimiento y la pérdida de agua a) y la pérdida de peso $b$ ).

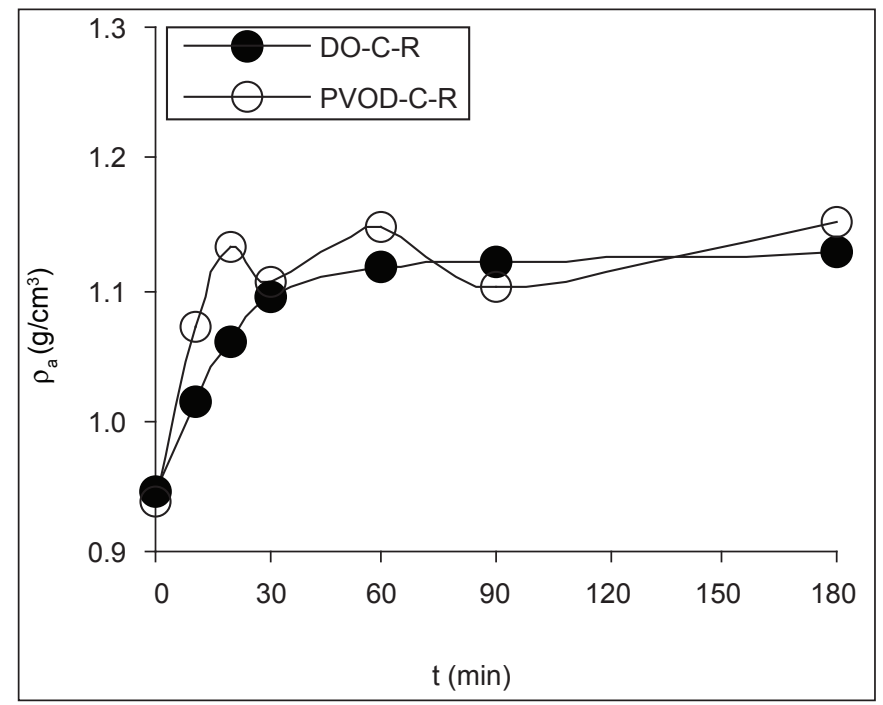

FIGURA 4 - Densidad aparente en función del tiempo para diferentes condiciones.

El cilindro fue la geometría que más perdió peso y agua, y a su vez obtuvo la menor ganancia de sólidos, debido probablemente a que es la geometría de menor área superficial. La aplicación del pulso de vacío, es recomendable para tiempos de proceso cortos, puesto que la velocidad de deshidratación en los tratamientos a PVOD es mayor que a OD. El promedio de la pérdida de peso en todos los tratamientos al final del proceso fue $27 \%$, para pérdida de agua fue $53 \%$, la ganancia de sólidos fue $25 \%$ y la pérdida de volumen de $50 \%$ y la máxima transferencia de masa ocurre antes de $30 \mathrm{~min}$. Existe una correlación lineal entre la pérdida de agua o la pérdida de peso y el encogimiento en las muestras sometidas a OD.

\section{5 - REFERENCIAS BIBLIOGRAFICAS}

[1] AOAC. Official methods of analysis. $13^{\text {th }}$ ed. Association of Official Analytical Chemist, Washington, USA, 1990.

[2] AZUARA, E.; BERISTAin, C. I.; GUTiÉRREZ, G. F. Osmotic dehydration of apples by immersion in concentrated sucrose/maltodextrin solutions. J. Food Process Preserv., v. 26, p. 295-306, 2002.

[3] BARAT, J. M. Desarrollo de un modelo de la deshidratación osmótica como operación básica. Valencia, España, 1998, 341 p. Tesis doctoral - Universidad Politécnica de Valencia.

[4] BERISTAIN, C. I.; AZUARA, E.; CORTÉS, R.; GARCÍA, H. S. Mass transfer during osmotic dehydration of pineapple rings. Int. Journal Food Sci. Tech., v. 25, p. 576-582, 1990.

[5] BONGIRWAR, D. R.; SREENIVASAN, A. Studies on osmotic dehydration of banana. J. Food Sci. Tech. v. 14, p.104-112, 1977.

[6] CORTÉS, J. H.; RIAÑO, C. E. Deshidratación Osmótica al vacío de pitahaya y mango. Cenicafé (Colombia), v. 46, n. 3, p. 129-139, 1995.

[7] EL-AOUAR, A. A; MOREIRA-AZUBEL, P.; XIDIEHMURR, F. E. Drying kinetics of fresh and osmotically pre-treated papaya (Carica papaya L.). J. Food Eng., v. 59, p. 85-91, 2003.

[8] FITO, P.; ANDRÉS, A.; CHIRALT, A.; PARDO, P. Coupling of hydrodynamic mechanism and deformation - relaxation phenomena during vacuum treatments in solid porous food liquid systems. J. Food Eng., v. 27, p. 229-240, 1996.

[9] GENINA, P. Deshidratación osmótica: Alternativa para conservación de frutas tropicales. Avance y Perspectiva (Colombia), v. 21, p. 321-324, 2002.

[10] KAWAMURA, T. Method of dehydrating foods. US Patent No. 4,788,072, 1988.

[11] LERICI, C. R.; PINNAVAIA, G.; DALLA ROSA, M.; BARTOLUCCI, L. Osmotic dehydration of fruit: influence of osmotic agents on drying behavior and product quality. J. Food Sci., v. 50, p. 1217-1219, 1985.

[12] MADAMBA, P. S.; LOPEZ, R. I. Optimization of the osmotic dehydration of mango (Mangifera Indica L.) slices. Drying Tech., v. 20, n. 6, p. 1227-1242, 2002.

[13] MELO, L. A.; ORDÓÑEZ, H.; LÓPEZ, O. B. Efecto de la presión y de la temperatura durante la deshidratación osmótica del mango Tommy Atkins en solución de sacarosa. Noos (Colombia), v. 9, p. 127-139, 1999.

[14] MENDOZA, R.; SCHMALKO, M. E. Diffusion coefficients of water and sucrose in osmotic dehydration of papaya. Int. J. Food Properties, v. 5, n. 3, p. 537-546, 2002.

[15] MOREIRA, R.; SERENO, A. M. Evaluation of mass transfer coefficients and volumetric shrinkage during 
osmotic dehydration of apple using sucrose solutions in static and non-static conditions. J. Food Eng., v. 57, p. 25-31, 2003.

[16] MORENO, J.; BUGUEÑO, G.; VELASCO, V.; PETZOLD, G.; TABILO-MUNIZAGA, M. Osmotic dehydration and vacuum impregnation on physicochemical properties of Chilean papaya (Carica candamarcensis). J. Food Sci., v. 69, n. 3, p. 102-106, 2004.

[17] MOYANO, P. C.; VEGA, R. E.; BUNGER, A.; CARRETÓN, J.; OSORIO, F. A. Effect of combined processes of osmotic dehydration and freezing on papaya preservation. Food Sci. Tech. Int., v. 8, n. 5, p. 295-297, 2002.

[18] MÚJICA-PAZ, H.; VALDEZ-FRAGOSO, A.; LÓPEZMALO, A.; PALOU, E.; WELTI-CHANES, J. Impregnation properties of some fruits at vacuum pressure. J. Food Eng., v. 56, p. 307-314, 2003.

[19] Nieto, A. B.; SAlvatori, D. M.; CASTRO, M. A.; ALZAMORA, S. M. Structural changes in apple tissue during glucose and sucrose osmotic dehydration: shrinkage, porosity, density and microscopic features. J. Food Eng., v. 61, p. 269-278, 2004.

[20] PALOU, E.; LÓPEZ-MALO, A.; ARGAÍZ, A.; WELTI, J. Osmotic dehydration of papaya. Effect of syrup concentration. Rev. Esp. Cienc. Tecnol. Aliment., v. 33, n. 6, p. 621-630, 1993.

[21] PARJOKO, K. A.; RAHMAN, M. S.; BUCKLE, K. A.; PERERA, C. O. Osmotic Dehydration kinetics of pineapple wedges using palm sugar. Lebensm Wiss Technol., v. 29, p. 452-459, 1996.

[22] POMPEU, G. A.; CAMPOS, P.; PAllet, D.; CASTle, $\mathrm{H}$. Use of a combined process of osmotic dehydration and deep-fat frying to obtain mango chips from cultivar Tommy Atkins. Acta Horticulture, v. 645, p. 285-291, 2004.

[23] RASTOGI, N. K.; RAGHAVARAO, K. S. M. S. Kinetics of osmotic dehydration under vacuum. Lebensm Wiss Technol., v. 29, p. 669-672, 1996.

[24] RASTOGI, N. K.; RAGHAVARAO, K. S. M. S.; NIRANJAN, K.; KNORR, D. Recent developments in osmotic dehydration: methods to enhance mass transfer. Trends in Food Sci. and Tech., v. 13, n. 2, p. 48-59, 2002.
[25] RODRIGUES, A. C. C.; CUNHA, R. L.; HUBINGER, M. D. Rheological properties and color evaluation of papaya during osmotic dehydration processing. J. Food Eng. v. 59, p. 129-135, 2003.

[26] SABLANI, S. S.; RAHMAN, M. S. Effect of syrup concentration, temperature and sample geometry on equilibrium distribution coefficients during osmotic dehydration of mango. Food Res. Int., v. 36, p. 65-71, 2003.

[27] SALVATORI, D.; ALZAMORA, S. M. Structural changes and mass transfer during glucose infusion of apples as affected by blanching and process variables. Drying Tech., v. 18, n. 1\&2, p. 361-382, 2000.

[28] SAPUTRA, D. Osmotic dehydration of pineapple. Drying Tech., v. 19, n. 2, p. 415-425, 2001.

[29] SERENO, A. M.; MOREIRA, R.; MARTÍNEZ, E. Mass transfer coefficients during osmotic dehydration of apple in single and combined aqueous solutions of sugar and salt. J. Food Eng., v. 47, p. 43-49, 2001.

[30] SHI, J.; LE MAGUER, M. Osmotic dehydration of foods: mass transfer and modeling aspects. Food Rev. Int., v. 18, n. 4, p. 305-335, 2002.

[31] SHYU, S.; HWANG, L. Effects of processing conditions on the quality of vacuum fried apple chips. Food Res. Int., v. 34, p. 133-142, 2001.

[32] TAPIA, M. S.; LÓPEZ-MALO, A.; CONSUEGRA, R.; CORTE, P.; WELTI-CHANES, J. Minimally processed papaya by vacuum osmotic dehydration (VOD) techniques. Food Sci. Tech. Int., v. 5, n. 1, p. 41-49, 1999.

[33] TORREGIANI, D. Osmotic dehydration in fruit and vegetable processing. Food Res. Int., v. 26, p. 59-68, 1993.

[34] VAN NIEUWENHUIJZEN, N. H.; ZAREIFARD, M. R.; RAMASWAMY, H. S. Osmotic drying kinetics of cylindrical apple slices of different sizes. Drying Tech., v. 19, n. $3 \& 4$, p. 525-545, 2001 .

\section{6 - AGRADECIMIENTO}

C.I. Ochoa-Martínez agradece a COLCIENCIAS (Instituto Colombiano para el Desarrollo de la Ciencia y la Tecnología Francisco José Caldas) por la beca-crédito para sus estudios doctorales. 